\title{
Não se deve identificar força explosiva com potência muscular, ainda que existam algumas relações entre ambas
}

\author{
Carlos Carvalho \\ Alberto Carvalho
}

https://doi.org/10.5628/rpcd.06.02.241

\author{
Laboratório do Movimento Humano \\ Instituto Superior da Maia \\ Portugal
}

\section{RESUMO}

O objectivo deste trabalho é procurar diferenciar a força explosiva da potência muscular. Apesar de ambas serem manifestações de força rápida, dependem de factores bastante diferentes, têm características distintas e necessitam de metodologias e métodos específicos e independentes no seu treino e desenvolvimento. Sumariamente, podemos dizer que o treino de força explosiva se deve realizar com cargas máximas e a máxima velocidade de contracção muscular, para que se garanta uma suficiente activação nervosa dos factores intramusculares (recrutamento, frequência e descarga dos impulsos e sincronismo das unidades motoras). Contrariamente, o treino da potência deve ser realizado com cargas médias, com máxima velocidade de execução de um determinado gesto, acção e/ou sequência motora específica. Daí cair fortemente na esfera do treino coordenativo ou técnico. Assim se depreende que sejamos da opinião de que estas duas manifestações de força rápida não podem, nem devem, ser identificadas como sinónimos de uma mesma capacidade motora.

Palavras-chave: força explosiva, potência muscular, treino da força.

\begin{abstract}
One should not identify explosive strength with muscular power even if some connection can be found between them

The aim of this paper is to search for the difference between explosive strength and muscular power. In spite of both being expressions of speed-strength, they depend on very different factors, have distinct characteristics and need specific and independent methods and methodologies. To sum it all up, it can be said that explosive strength training should be done with maximum loads and the maximum rate of velocity of muscular contraction so that a sufficient nervous activation can be achieved in the intramuscular factors (recruitment, firing frequency and synchronism of nervous stimulus). Contrarily to this, power should be trained with medium loads but with high speed of execution of any specific motor action and/or sequences, therefore falling on a more technical or coordinative training. We can deduce from all this that these two expressions of speed-strength should not be identified as synonyms of the same physical abilities.
\end{abstract}

Key Words: explosive strength, muscular power, strength training. 


\section{INTRODUÇÃO}

Desde há muito que defendemos que os equívocos terminológicos e conceptuais são questões limitativas da correcta compreensão e análise crítica dos fenómenos. Pensamos mesmo que, se os equívocos e as incorrecções não forem esclarecidos, dificilmente nos podemos entender com clareza e, pior do que isso, dificilmente se poderão conceber metodologias de treino de efectiva eficácia prática. Os conceitos de força explosiva e de potência muscular são, sem dúvida, dois exemplos paradigmáticos da falta de rigor, de consistência e de delimitação entre distintos tipos de força que vulgarmente encontramos na literatura da especialidade.

Assim, o objectivo deste trabalho é procurar diferenciar a força explosiva da potência muscular. Apesar de ambas serem manifestações de força rápida, dependem de factores bastante diferentes, têm características distintas e necessitam de metodologias e métodos específicos e independentes no seu treino e desenvolvimento.

\section{FORÇA RÁPIDA}

Como é sabido, devemos entender por força máxima (Fmax) o valor mais elevado de força que o sistema neuromuscular é capaz de produzir, independentemente do factor tempo (15). Logo que a força é condicionada pelo factor tempo entramos no "reino" da força rápida (Frap). A grande maioria das actividades desportivas depende não tanto de altas expressões de força, mas muito mais de que essa força ou parte dela se produza com elevada rapidez. Isto é verdade não só nos lançamentos, saltos ou remates, mas em todas as situações em que é necessário alterações rápidas de direcção e/ou aceleração, como acontece na grande maioria dos eventos desportivos (p. ex.: no futebol, andebol, voleibol, basquetebol, ginástica, etc.).

Se, para além do registo da força produzida, avaliarmos também o tempo do movimento, observamos uma correlação negativa entre Fmax e o tempo de movimento. Esta correlação aumentará à medida que as cargas se aproximam do máximo individual (15) e, por outro lado, se a resistência a vencer for baixa, a influência da Fmax diminui gradualmente e a velocidade de execução tenderá a assumir maior predominância.
Esta relação de força tempo (velocidade) pode ser expressa através da curva força-tempo $(\mathrm{C} \mathrm{f-t})$, ou, então, esta relação que se manifesta entre a força e velocidade pode ser representada por uma curva (hiperbólica) que denominamos por curva forçavelocidade ( $\mathrm{C} \mathrm{f-v}$ ). Ambas as curvas procuram expressar esta relação difícil, e aparentemente contraditória, de duas propriedades mecânicas dos músculos, que, a nível do rendimento se procura maximizar, a saber: (1) melhorar a capacidade de desenvolver mais força em menos tempo e (2) conseguir que as estruturas musculares tenham a mais elevada produção de força perante um aumento de velocidade de encurtamento muscular.

Como podemos observar na figura 1 , qualquer modificação que se produz na $\mathrm{C}$ f-t reflecte-se na $\mathrm{C} \mathrm{f-v} \mathrm{e}$ vice-versa. As modificações positivas na $\mathrm{C} \mathrm{f-t} \mathrm{(figura}$ 1a) produzem-se quando a curva se desloca para a esquerda e significam que: (i) para produzir a mesma força se tem menos tempo, ou que (ii) com o mesmo tempo se alcança mais força. Se os resultados são expressos através da $\mathrm{C}$ f-v as modificações positivas produzem-se quando a curva se desloca para a direita e o que se identifica é que (i) a mesma resistência desloca-se em maior velocidade, ou que (ii) à mesma velocidade desloca-se mais resistência (figura $1 \mathrm{~b}$ ).
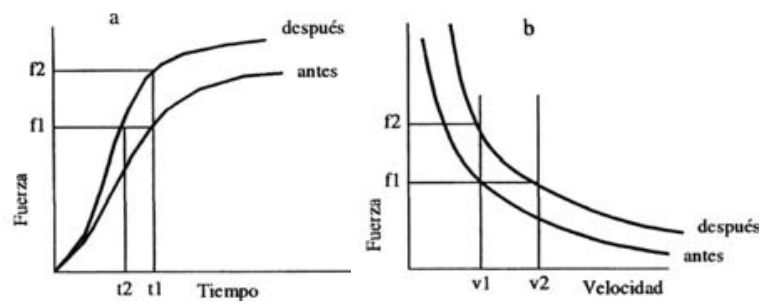

Figura 1. As alterações produzidas na $C f$-t são equivalentes às produzidas na $C f$-v [1].

No fundo, o problema da Frap é procurar harmonizar duas capacidades motoras que, em valores absolutos, se manifestam em oposição. Tal como se pode constatar pela análise da curva de Hill $^{1}$ (curva de forçavelocidade), quanto maior é a velocidade de acção muscular concêntrica, menor terá de ser a resistência a superar (no extremo, será sem carga, resistência $=$ 0 ), ou quanto maior a resistência a vencer, mais 
baixa será a velocidade manifestada (no extremo, a velocidade $=0$, acção estática/isométrica). Entre estes dois extremos, existe todo um espectro de possibilidades que, dependendo da carga a vencer, do tempo disponível para desenvolver força e do nível de velocidade de execução atingido, nos permite identificar diferente expressões de força rápida que vamos estruturar em dois grandes sub-grupos: da força explosiva e da potência.

\section{FORÇA EXPLOSIVA}

A Força explosiva (Fexp) é o resultado da relação entre a força produzida (manifestada ou aplicada) e o tempo necessário disponível. Portanto, a Fexp é a produção de força numa unidade de tempo e expressa-se em N.s-1. Podemos de uma forma mais perceptível entender e relacionar a força desenvolvida por um sujeito e a sua relação com o tempo através da análise atenta da $\mathrm{C} \mathrm{f-t} \mathrm{(figura} \mathrm{2).} \mathrm{Ao} \mathrm{contrário} \mathrm{da}$ manifestação máxima de força, enfatiza-se agora que essa Fmax ou percentagem seja atingida em cada vez menos tempo. Este conceito é materializado e avaliado pela subida (declive) mais ou menos íngreme da linha de força na curva f-t. Este súbito incremento de força era designado por Verschoshanskij, desde o início dos anos setenta, por força explosiva e corresponde, grosso modo, à mais rápida manifestação de força no mínimo tempo. Na literatura da especialidade, é denominada por Taxa de Produção de Força (TPF), o que significa "proporção, taxa ou velocidade de desenvolvimento ou produção de força em relação ao tempo" $(4,8)$.

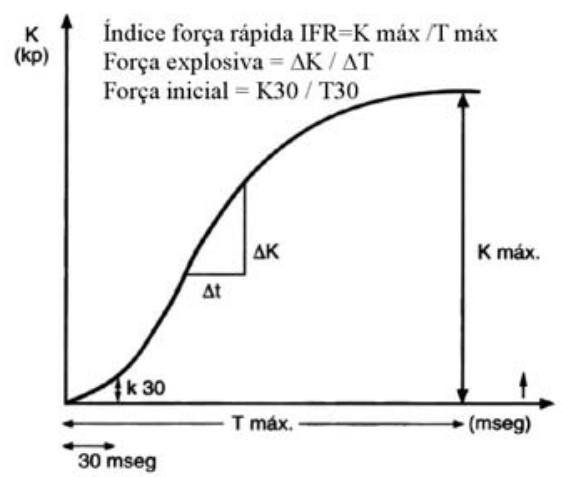

Figura 2. Curva f-t durante uma contracção isométrica que se referencia e formula em expressão matemática, respectivamente, Índice de Força Rápida, Força Explosiva e Força Inicial [3]
Esta expressão de força mede-se desde o início da manifestação de força até qualquer ponto da curva ft. Se se mede desde o início da produção de força até ao momento em que se alcança o valor mais elevado de força [Pico Máximo de Força $(P M F)$ ] e se esta acção se fizer contra carga máxima (de preferência contra uma carga inamovível), medimos o que Bürhle $(2,99)$ denomina de Índice de Força Rápida (I Fráp) e podemos definir por "Coeficiente entre o valor máximo de força e o tempo máximo que é necessário para o valor de Fmax ser atingido". É quantificado pela seguinte expressão matemática:

\section{Frap $=$ Fmax $/$ tmax}

Este índice de Fráp exige, nomeadamente na sua maior magnitude, muito tempo para ser atingido mais de 700 milésimos de segundos (ms) - o que é muito pouco característico da grande maioria das acções desportivas, em que o tempo disponível se situa, genericamente, por volta dos $250 \mathrm{~ms}$. É, por outro lado, neste período temporal que se manifestam as maiores expressões de Fexp. É aqui que a força explosiva é máxima (Fexp max ou TPFmax), expressa no declive mais acentuado da linha de força na curva de $\mathrm{f}$-t e quantificada matematicamente na equação Fexp $\max =\Delta F / \Delta t$. Por ser esta a sub-divisão de força que nos interessa particularmente, iremos, posteriormente, fazer uma análise mais detalhada. Existem alguns autores que, de acordo com este critério tempo, ainda referem uma outra manifestação de força que denominam por Força Inicial $(3,15,16)$. Definem-na pela capacidade de nos momentos iniciais da tensão muscular se desenvolver a mais alta expressão de força. É fundamental para o óptimo rendimento em disciplinas em que os gestos desportivos requeridos são de grande velocidade inicial, como o Boxe, Karaté, Esgrima, ou em todas as actividades onde haja somente um tempo disponível reduzido e em que seja importante obter consideráveis níveis de força. A Força Inicial é avaliada através do valor de força que é alcançado nos primeiros 30 ms $(2,3)$ ou 50 ms (15). Quando a resistência a vencer é muito pequena (inferior a $25 \%$ da Fmax) e o movimento a realizar pode ser considerado de natureza balística, o factor predominante é Força Inicial, também denominado por Taxa Inicial de 
Produção de Força (TIPF). Assim, por Força Inicial entende-se a capacidade de o sistema neuromuscular acelerar o mais rapidamente possível, desde zero $(14,16)$. Na curva força-tempo, a TIPF é o início do declive da curva (figura 2).

\section{FORÇA EXPLOSIVA MÁXIMA OU TAXA DE PRODUÇÃO DE FORÇA MÁXIMA}

Zatsiorskij, citado por Harre e Lotz (10), define força explosiva como a capacidade de obter valores elevados de força em tempo muito curto. O mesmo quer dizer que é a capacidade neuromuscular de superar com alta velocidade de execução resistências bastante altas. A força explosiva depende da velocidade de contracção muscular, em resultado da estimulação neuro-sensorial, ou seja, depende fortemente da intensidade da descarga dos impulsos nervosos $(5,7,8,9,12)$.

Havendo uma infinidade de possibilidades de medições da força explosiva (Fexp) entre os diferentes pontos da curva f-t, constata-se, no entanto, que existe um momento em que a produção de força por unidade de tempo é a mais elevada de todo o percurso da curva, e essa produção de força denomina-se de força explosiva máxima (Fexp max) ou Taxa de Produção de Força Máxima (TPFM). Podemos definila pela máxima produção de força por unidade de tempo em todo o percurso de produção de força. É medida, geralmente, em situação de força isométrica ou pelo menos na fase estática de uma acção dinâmica, e, por isso mesmo, a Fexp max produz-se no início da produção de força, i.e., nos primeiros $100 \mathrm{~ms}$, sendo aqui que encontramos a fase de máximo declive da curva f-t.

Se medirmos a força dinamicamente, como se pode observar na figura 3, o pico máximo de força $(P M F)$ diminui gradualmente à medida que reduzimos a resistência; quanto mais rapidamente se executa o movimento, menor é o tempo de intervenção, mas, genericamente, é idêntico o nível de desenvolvimento de força. Isto constata-se pela forma de subida ou evolução da curva de força. Também sabemos o que acontece se as cargas a vencer forem de cada vez mais baixa magnitude: o nível de produção diminui irremediavelmente. Assim, com resistências inferiores a $30 \%$ da Fmax (isométrica), a inclinação da linha da curva de f-t declina para a direita. É, no entanto, comummente aceite que, com resistências superiores a $30 \%$ da força máxima, a Fexp max é estável e pode ser sempre máxima.

Segundo Badillo e Serna (1), se a resistência a vencer for inferior a $30 \%$ da Fmax, quase imediatamente após o início de aplicação da força começa a existir movimento; o corpo começa a mover-se antes de se ter aplicado a força necessária para produzir o máximo de força, pelo que não se consegue alcançar o valor máximo de força explosiva Fexp, já que o corpo começa a deslocar-se e a força aplicada por unidade de tempo é tanto menor quanto maior for a velocidade de deslocamento. De tudo isto se deduz facilmente que a força explosiva máxima se produz na fase estática de qualquer deslocamento de uma resistência e que se a resistência for muito pequena não se pode produzir a dita força explosiva máxima $(1,22)$. Por outro lado, há autores que referem que quanto mais elevada for a velocidade, menor é a expressão de força, porque as conexões dos filamentos de actinamiosina se fazem de forma cada vez mais débil, logo é menor a tensão desenvolvida pelo músculo $(5,13)$.

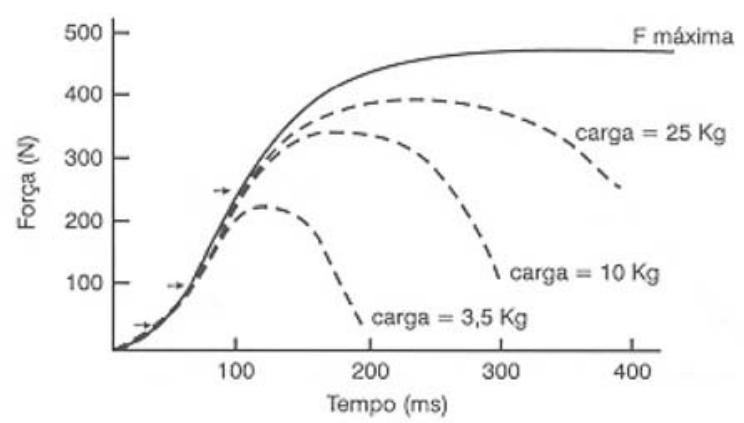

Figura 3. Curva f-t para aç̧ão isométrica e concêntrica contra diferentes cargas, em movimento de braço no lançamento de peso de um sujeito. As setas indicam o início da fase concêntrica dos diferentes movimentos [16].

Isto leva-nos, de momento, a duas reflexões. Primeira, que a Fexp max não tem nada ou tem pouco que ver com o movimento, mas sim com a sua produção, e segunda, que se deduz da primeira, que é o facto de não fazer grande sentido associar a Fexp Max, unicamente, com movimentos muito rápidos. Os exercícios explosivos (ou acções explosivas) não são os que são produzidos a grande velocidade, mas sim aqueles em que se alcança a máxima ou quase máxima produção de força em uma unidade 
de tempo $(16,387)$. Obviamente que a Fexp e a Fexp max têm uma estreita relação com a velocidade de movimento perante uma determinada resistência, já que a maior ou menor velocidade depende precisamente da capacidade de produzir força rapidamente. Quanto maior é o grau de desenvolvimento da força (produzido na fase estática) mais rapidamente pode ser realizada a fase de aceleração (fase que começa precisamente no início do movimento) (18).

A Fexp e a Fexp max, como referimos, conseguem maiores expressões de força em presença de resistências máximas ou quasi-máximas. Logo, perante cargas de tal magnitude, para além de existir uma fase estática considerável, a velocidade de deslocamento terá de ser sempre reduzida. A sua aceleração é, naturalmente, baixa, no entanto, a "intenção" de vencer essa carga tem de ser feita com grande explosividade e, assim, a velocidade de contracção muscular terá de ser máxima.

Há aqui que distinguir dois conceitos que muitas vezes a literatura da especialidade não tem explicitado convenientemente: uma coisa é a velocidade de execução de um movimento e outra é a velocidade de contracção muscular, que, não sendo antagónicas, no caso de cargas de magnitude considerável, não são coincidentes.

É por isso que os métodos da Taxa de Produção de Força, também designados de Métodos Máximos, têm como objectivo incrementar a força explosiva e fazemno através do aumento da capacidade de activação nervosa. Por aumento da capacidade de activação nervosa devemos entender todo o conjunto de mecanismos neurais que podem contribuir para aumentar a capacidade de o músculo produzir força, nomeadamente o recrutamento, a frequência de actividade e a sincronização das unidades motoras (UMs).

Se as UMs que têm a capacidade de produção de força mais elevada são as UMs da fibra tipo II, de acordo com o Princípio do Recrutamento das UMs, estas só serão recrutadas se a resistência a vencer for suficientemente grande para que o limiar de recrutamento seja atingido. Por esta razão, para mobilizar as fibras rápidas, é necessário vencer resistências muito próximas do máximo individual, pois só assim se garante o recrutamento dessas fibras. Complementarmente, para solicitar o aumento da frequência de actividade das UMs, i.e., o número de estímulos por unidade de tempo, é crucial que a acção muscular seja realizada de forma explosiva, logo, com uma grande velocidade de contracção muscular. E é aqui que reside o cerne do treino com objectivos de adaptação neural. Assim, é no cumprimento desta dialéctica que se alicerçam todos os métodos de treino da força explosiva ou, também denominados, métodos de desenvolvimento da Taxa de Produção de Força.

Por este motivo o treino de Fexp e de Fexp max deve ser realizado, preferencialmente, com cargas máximas ou próximas do máximo e com uma velocidade explosiva de contracção muscular. Tendo em conta a capacidade de produzir força, o tempo disponível e resistência a vencer, há que ajustar o treino à especificidade dos gestos e acções desportivas que se pretende desenvolver.

Para terminar, é importante referir que a Fexp depende dos seguintes factores principais: capacidade de Fmax, tipo e características de contracção das fibras musculares e coordenação intra-muscular (recrutamento, frequência da descarga de impulsos e sincronismo das UMs), e que estes factores se reflectem na produção rápida de força na fase estática e início do movimento e na velocidade de encurtamento do músculo.

\section{POTÊNCIA MUSCULAR}

De volta à Potência Muscular iremos começar por fazer uma abordagem sintética da sua perspectiva mecânica e procurando, de seguida, aproximarmonos da sua dimensão de capacidade motora.

O conceito mecânico de trabalho (W) refere o produto da força aplicada a um corpo pela distância que percorre na direcção da sua aplicação (W=F.d). Expressa-se em Newton.metros (N.m) ou em Joules (J), (1 J = 1 N.m).

Por Potência $(\mathrm{P})$ entende-se a razão entre um determinado trabalho mecânico e o tempo em que é efectuado ( $\mathrm{P}=\mathrm{W} / \mathrm{t}$ e expressa-se em Joules/seg. ou Watts).

Se a velocidade é o espaço percorrido em determinado tempo $\left(\mathrm{m} \cdot \mathrm{s}^{-1}\right)$, teremos então que $\mathrm{P}=\mathrm{W} / \mathrm{t}$ o que poderemos substituir por $\mathrm{P}=\mathrm{F}$. d. $\mathrm{t}^{-1}$. Daí podermos formular que a Potência é igual ao produto da Força pela Velocidade $(\mathrm{P}=\mathrm{F} . \mathrm{V})$, ou seja, o produto da Força que um segmento do corpo pode produzir pela velocidade desse segmento. 
O conceito de potência é, deste modo, importante para o treino e está, naturalmente, associado à curva f-v. A potência será, assim, o produto da força pela velocidade em cada instante do movimento. Existe também uma curva de potência, dependente da curva f-v. Ou, mais importante ainda, é o melhor produto força-velocidade conseguido através do movimento que determina o pico máximo de potência, e é este que define as características dinâmicas da força aplicada durante um exercício.

Tendo em atenção o que é evidenciado na curva força-velocidade expressa pela curva em forma de hipérbole de Hill, representada na figura 4, nota-se que, quanto mais elevada é a carga a vencer, mais força tem de ser produzida pela componente contráctil e menor é a velocidade de encurtamento dessas componentes musculares; o ponto mais elevado desta sequência corresponde à força máxima isométrica (estática), já que a velocidade deixa de acontecer, como já referimos. Neste ponto a potência é também nula ( $\mathrm{P}=$ Fmáx .0$)$. No outro extremo da curva, joga-se uma situação inversa em que a carga é igual a zero. O músculo movimenta-se livremente, a velocidade é máxima, pelo menos teoricamente, voltando a Potência a ser nula ( $\mathrm{P}=0 . \mathrm{Vmáx})$. Desta forma surge a curva da potência em forma de sino que oscila entre dois limites, procurando compatibilizar dois factores que conceptualmente se opõem mutuamente (figura 4).

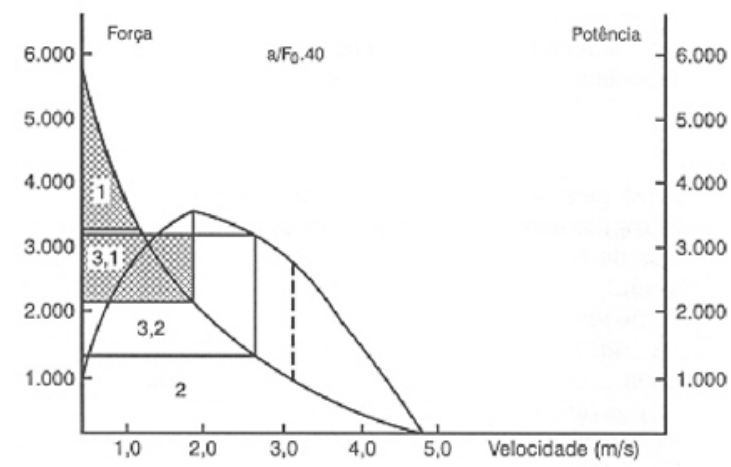

Figura 4. Partindo do pressuposto de que quanto mais elevada for a carga a vencer mais força tem de ser desenvolvida e menor é a velocidade de encurtamento muscular, poder-se-á formar uma curva que oscila entre dois limites, em forma de sino, que procura compatibilizar estes dois factores (forçavelocidade) (Adaptado de 17).
A maior potência e a velocidade máxima de contracção não se conseguem perante resistências ligeiras, nem quando utilizamos grandes resistências a velocidade baixa, mas quando realizamos o movimento com cargas e com velocidades intermédias.

Segundo a figura 4, Tihanyi (17), a curva força velocidade e de potência será um contínuo em que se distinguem três grandes zonas:

1 - Zona de influência de força maximal: onde se utiliza máxima ou grande força e mínima ou pouca velocidade. A potência desenvolvida é média ou baixa.

2 - Zona de velocidade maximal: consegue-se uma grande velocidade perante resistências pequenas. A potência será também média ou baixa.

3 - Zona de potência, em que a força aplicada e a velocidade apresentam valores intermédios. A potência alcança os seus níveis máximos e está ainda subdividida:

3.1 - Parte em que o cume do rendimento é limitado pela força máxima;

3.2 - Parte em que o cume do rendimento é limitado pela velocidade máxima.

Poderemos interpretar que nesta zona 3 dever-se-á treinar, ao mesmo tempo, a força e velocidade na sua expressão maximal. Esta zona joga, assim, um papel determinante do ponto de vista do desenvolvimento da força e velocidade.

Antigamente chamava-se a atenção para o facto de que esta relação não se podia fazer seguramente. Agora esta relação é claramente assegurada pelo consenso dos resultados de investigação, os quais demonstram que o treino da força move, favoravelmente, a curva força-velocidade para a direita. Depois do treino da Força, o músculo é mais forte em todas as velocidades de movimento desde uma contracção isométrica a uma contracção realizada à velocidade máxima (6).

O incremento da potência máxima é sempre positivo para o desportista, ainda que a melhoria possa ser gerada por vias distintas e com resultados também distintos. Quando se trabalha com cargas ligeiras, a melhoria da potência consegue-se perante carga idêntica pelo aumento da velocidade de execução; mas quanto maior for a carga aplicada, a melhoria, se se produzir, terá lugar pela magnitude das cargas utilizadas, o que significa que terá havido um 
aumento da força e, provavelmente, também de velocidade. O objectivo do treino e as necessidades de cada especialidade devem marcar a via mais adequada das melhorias.

Os valores concretos de força e velocidade (supõe-se que estas sejam sempre o máximo possível), nos quais se alcançam a potência máxima não são os mesmos em todos os sujeitos e especialidades. Em termos médios, a força (resistência a vencer) deve estar entre os 30 a $40 \%$ da força isométrica máxima e a velocidade entre os 35 e os $45 \%$ da velocidade máxima de contracção contra resistências muito ligeiras ou nulas $(5,17)$. A oscilação dentro destas margens dependerá das características do desportista e do tipo de treino realizado. Os indivíduos mais fortes e/ou mais lentos geralmente conseguem a sua potência máxima a velocidade menor do que os mais rápidos.

Como resultado, os factores que afectam tanto a força muscular quanto a velocidade de encurtamento determinarão a potência que pode ser produzida. Quando a um músculo chega um impulso nervoso adequado, segundo Enoka (5, p. 307), "os principais determinantes da produção de potência são o número de fibras musculares activadas em paralelo e a velocidade com que os miofilamentos podem converter energia em trabalho mecânico". Embora a produção de potência seja máxima quando a força muscular é de cerca de um terço do máximo, a produção de potência aumenta à medida que o músculo se torna mais forte (a área de secção transversal aumenta), e, assim, o valor de um terço aumenta (5). E daqui, desde logo, podemos depreender quão errada é, do ponto de vista do treino e incorrecta na perspectiva biomecânica, a convicção de que o desenvolvimento da força muscular induz perda de velocidade.

Por outro lado, a expressão de um movimento desportivo implica, normalmente, a execução de gestos e/ou de um conjunto de gestos com que se procura a harmonização e optimização da acção de um conjunto de músculos que têm responsabilidade na concretização dessa acção. Assim, uma outra possibilidade para o aumento da potência muscular decorre da melhoria da coordenação intermuscular. A coordenação intermuscular expressa a capacidade da melhor cooperação possível entre os músculos agonistas, antagonistas e sinergistas, no que diz respeito ao objectivo do movimento em causa. A melhoria da potência muscular, que pode atribuir-se a uma melhor coordenação intermuscular, está muito dependente da especificidade do movimento, o que significa que não é muito transferível de um movimento/gesto para outro.

De facto, é bem conhecido que o desenvolvimento de força é específico de determinado padrão de movimento, de determinada velocidade e de tipo de acção muscular utilizados durante o treino. Isto é, naturalmente, ainda muito mais evidente no treino para melhoria de potência, que, na prática, exige domínio de movimentos complexos com interferência de um conjunto de músculos geralmente multi-articulares. Só podemos, então, treinar potência, como de resto velocidade, após um domínio perfeito do gesto que pretendemos desenvolver. Quanto melhor for a mestria técnica do gesto, mais veloz e perfeita a execução e, com isso, o produto final pretendido.

De facto, o treino da potência é, antes de tudo, um aprimoramento, entre duas importantes qualidades: força e velocidade. Donde o treino da potência tem de ser efectuado com uma carga leve $(30-60 \%$ da carga máxima de acordo com exigência do gesto desportivo pretendido) e vencida à máxima velocidade de execução a tal ponto que, como Schmidtbleicher gosta de caricaturar, seria possível ouvir o "silvar" do movimento; nessa medida, o treino cai fortemente no âmbito do treino coordenativo específico ou treino técnico.

\section{EM SÍNTESE}

A força explosiva e a potência devem enquadrar-se no grupo das manifestações da força muscular que, geralmente, denominamos de força rápida. Ambas são condicionadas, por esse motivo, pelo factor tempo em que se realizam ou se devem realizar. No entanto, para a força explosiva o determinante é o nível de força expressa e o tempo necessário para tal, independentemente de existir ou não movimento; na potência, o factor substantivo é a velocidade com que se consegue vencer uma determinada resistência, por isso, a sua expressão máxima corresponde à conjugação óptima da carga a vencer (ca. $40 \%$ da força máxima isométrica) e da velocidade máxima de movimento que se consegue alcançar perante tal carga. Inferindo-se daí que o treino de força explosi- 
va deve realizar-se com cargas máximas e à máxima velocidade de contracção muscular, para que se garanta uma suficiente activação nervosa dos factores intramusculares (recrutamento, frequência e descarga dos impulsos e sincronismo das unidades motoras). Contrariamente, o treino da potência deve ser realizado com cargas médias, com máxima velocidade de execução de um determinado gesto, acção e/ou sequência motora específica (cooperação óptima entre os músculos agonistas, antagonistas e sinergistas); daí cair fortemente na esfera do treino coordenativo ou técnico.

\section{NOTAS}

${ }^{1}$ Deixando-se contrair um músculo quando parte de idêntico comprimento mas contra diferentes magnitudes de carga, registam-se diferentes velocidades de encurtamento, o que se denominou por relação força-velocidade. Esta relação deixa-se muito bem descrever por uma função hiperbólica que Hill (11) transformou num expressão matemática que é conhecida por equação de Hill.

\section{BIBLIOGRAFIA}

1. Badillo JJG, Serna JR (2002). Bases de la Programación del entrenamiento de fuerza. Rendimiento Deportivo. INDE Publicaciones. España.

2. Bührle M. (1985). Dimension des Kraftverhaltes und ihre spezifischen Trainingsmethoden. In M. Bührle (Hrsg.) Grundlagen des Maximal-und Schnellkrafttrainings.

Schriftenreihe des Bundsinstitut für Sportwissenschaft 56. Schorndorf: Hofman, 82-111.

3. Bührle M (1986). Zum Grundkonzept des Kraft-und Sprungkrafttrainings. In Carl K, Schiffer J (eds.) Zur Praxis des Sprungskrafttrainings. Bundesinstitut Sportwissenschaft.

4. Bührle M, Schmidtbleicher D (1981). Komponenten der Maximal und Schnellkraft-Versuch einer Neustrukturierung auf der Basis empirischer Ergebnisse. Sportwissenschaft 11, 11-27.

5. Enoka RM (2000). Bases Neuromecânicas de Cinesiologia (2a Edição). Brasil: Manole.

6. Fleck SJ, Kraemer WJ (1987). Designing Resistance Training Programs. Champaign, Il.: Human Kinetics Books.

7. Häkkinen K (1985). Factors influencing trainability pf muscle strength during short term and prolonged training. National Strength and Conditioning Association Journal 7: 32-37.

8. Häkkinen K, Komi PV (1983). Electromyographic changes during strength training and detraining. Medicine and Science in Sports and Exercise 15: 455-460.

9. Häkkinen K, Komi PV (1985). Effects of explosive type strength training on electromyographic and force production characteristics of leg extensor muscles during concentric and various strech-shortening cycle exercises. Scand $J$ Sports Sci 7, 65-76.

10. Harre D, Lotz L (1989). O treino da força rápida. Revista Treino Desportivo, 12.

11. Hill AV (1938). The heat of shortening and dynamic constraits of muscle. Proceedings of the Royal Society of London. B126: 136-195.

12. Sale DG (1992). Neural adaptation to strength training. In Komi P (ed.) Strength and Power in Sport. Oxford: Blackwell Science, 381-395.

13. Sale DG, Norman RW (1982). Testing Strength and Power. In MacDougall et al. (eds.) Physiological testing of the elite athletes. New York: Movement Publ., Inc., 7-37.

14. Schmidtbleicher D (1984). Strikturanalyse des motorischen Eigenschaft. In Krafttraining Lehre des Leichtathletik. Beilage zur zeitschrift Leichathletik 35(50): 1785-1792.

15. Schmidtbleicher D (1985). Klassifizierung des Trainingsmethoden. In Krafttraining Lehre des Leichtathletik. Beilage zur zeitschrift Leichathletik 35(50): 1785-1792.

16. Schmidtbleicher D (1992): Trainings for power events. In Komi, P. (ed.) Strength and Power in Sport. Oxford, Blackwell Science, 381-395.

17. Tihanyi J (1988). Prinzipien individualisierter Trainings protokolle auf der Basis der Muskel-faserzusammensetzung und mechanischer Merkmal. Leistungssport 2, 41-45.

\section{CORRESPONDENCIA}

\section{Carlos Carvalho}

Laboratório do Movimento Humano

Instituto Superior da Maia

Av. Carlos Oliveira Campos

4474-690 Avioso, S. Pedro

Portugal

ccarvalho@ismai.pt
18. Verkhoshansky Y (1996). Componenti e structtura dell,impegno esplosivo di forza. Rivista di Cultura Sportiva ano XV, n. 34: 15-21. 